\title{
Fine grained concrete structures reliability assessment: theory and investigational studies
}

\author{
Sergey Parfenov ${ }^{1}$, Anatoly Alekseytsev ${ }^{2, *}$ and Yuriy Vinokurov ${ }^{1}$ \\ ${ }^{1}$ Bryansk State Engineering and Technology University, Stanke Dimitrov av., 3, Bryansk, 241037, \\ Russian Federation \\ ${ }^{2}$ Moscow State University of Civil Engineering, Yaroslavskoe shosse, 26, Moscow, 129337, Russia
}

\begin{abstract}
Describes the theoretical preconditions of using mechanical properties of fine-grained concrete in the design of concrete structures subject to risks and the general safety. A technique for experimental studies and data on the fine-grained concrete deformative properties at different ages and different loading levels are presented. The regularities of the modulus of elasticity change from strength, type and age of concrete are revealed. Full diagrams of deformation of concrete are constructed. The results obtained can be used in the design of the fine-grained concrete structures in buildings having risks occurrence socioeconomic losses and able to resist of emergency actions.
\end{abstract}

\section{Introduction}

Currently, designing the structures with regard to their reliability requirements appears challenging. Many authors have studied the reliability of structures made of various materials. For example, the reliability of reinforced concrete $[1-3,13]$ and steel $[4,5,10]$ structures of various types has been investigated. Methods for design solutions optimization $[7,10]$ have been developed, which do not always take into account the probability of structure failure. Thus, on approaching the optimal solution, we also increase the risk associated with emergency situations. To prevent such effects, both theoretical $[6,8,11,13,14]$ and experimental [12] studies are carried out. The issues estimating the dispersion of mechanical characteristics of concrete and reinforced concrete structures are particularly important in this case. At the same time, in the process of designing the reinforced concrete structures, not only the strength properties but also deformative concrete properties are taken into account that are to a considerable extent determined by concrete elasticity modulus $E_{\delta}$ by the ratio of elastic and inelastic deformations at various loading levels, by limiting concrete deformations at compression $\varepsilon_{\delta}^{\mathrm{lim}}$. In this article, the values of concrete mechanical characteristics are determined experimentally by its age and loading level.

\footnotetext{
* Corresponding author: aalexw@mail.ru
} 


\section{Methods}

The value $R_{D}$ of economic and social loss risk is broadly determined in the following way:

$$
R_{D}=\sum_{i=1}^{n}\left(\left(U_{1}+U_{2}\right) \cdot p\left(R_{p}, \eta\right)\right),
$$

where $n$ - the number of structures for which the possibility of transition to disrupted condition is considered; $U_{1}$ - material damage in case of structure failure; $U_{2}$ - possible social losses in monetary terms. The function $p\left(R_{p}, \eta\right)$ - probability of structure failure during normal operation, determined by compression resistance $R_{p}$ and loading level $\eta$.

Value of $p\left(R_{p}, \eta\right)$ is determined in the following way [8]:

1. Calculate the load capacity $\Delta$ and mean square deviation $\Omega$ :

$$
\Delta=\bar{Q}-\bar{R}_{p}, \Omega=\sqrt{S_{Q}^{2}+S_{R_{p}}^{2}},
$$

where $\bar{Q}$ - mean loading effect; $\bar{R}_{p}$ - the average value of the standard resistance for this effect; $S_{Q}, S_{R_{p}}$ - dispersions of these values under consideration.

2. Probability $p\left(R_{p}, \eta\right)$ value is estimated:

$$
p\left(R_{p}, \eta\right)=1-(0,5+\Phi(\Delta / \Omega)),
$$

where $\Phi$ - Laplace's function.

Damage value $U_{1}$ is calculated as per equation:

$$
U_{1}=U_{1 m a t}+U_{1 m o n t}+U_{1 \text { rep }},
$$

where $U_{1 \text { mat }}$ - element material cost; $U_{1 \text { mont }}$ - assembly tooling cost, $U_{1 \text { rep }}$ - element reconstruction cost. The cost of social loss in failure of some element can be calculated in the following way:

$$
U_{2}=\bar{N} U_{P},
$$

where $\bar{N}$ is the average count of people working or happened round the area of destruction in emergency onset; $U_{P}$ is the average amount of compensation per one person, as determined by law [9].

Define the procedure for the experimental determination of $R_{p}$ value for fine-grained concrete. The ratio of the elastic $\varepsilon_{y}$ and inelastic $\varepsilon_{\delta}$ concrete deformations are characterized by the elasticity coefficient $v$ and the plasticity coefficient $\lambda$.

$$
v=\frac{\varepsilon_{y}}{\varepsilon_{\delta}}=1-\lambda,
$$

For practical calculations of reinforced concrete structures, the introduced average concrete elasticity modulus is used:

$$
E_{\delta}^{\prime}=\frac{\sigma_{\delta}}{\varepsilon_{\delta}}=E_{\delta} \frac{\varepsilon_{y}}{\varepsilon_{\delta}}=E_{\delta} v
$$

It follows from relation (7) that the elasto-plasticity modulus is a variable value depending on the loading level. 
The elastic modulus was experimentally calculated as per GOST 24452-80 for each sample at $30 \%$ loading level of the destructive loading, according to the formula:

$$
E_{\delta}=\frac{\sigma_{\delta}}{\varepsilon_{y}}
$$

Within the loading stage, deformations were determined by the linear interpolation. Elasticity and plasticity coefficients were calculated according to the formula (6) for all prisms, and also the average elasto-plasticity modulus was calculated in expression (7) at the loading level $\eta=\sigma_{\delta} / R_{p}=0,5$.

\section{Results and discussion}

In course of the study, deformations were determined on concrete prism-specimens having

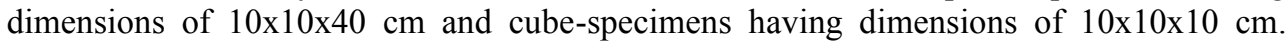
These specimens were prepared in separate lots from one batch. Each series consisted of 24 prisms and 24 cubes. Cubes were designed to determine the strength over time. The prisms were designed to determine shrinkage strains ( 3 pcs.), prismatic strength, elastic modulus at the age of 14 and 28 days ( 6 pcs.), tensile strength in bending and splitting ( 3 pcs.), creep strains at the age of 14 and 28 days at different loading levels (12 pcs.). For comparison with normal-weight (NW) concretes, V.S. Kuznetsov's data ( $\bar{R}$ in table 1) [6] were involved.

When comparing the average experimental values of the elasticity coefficients in Table 1 , it was found that at low loading levels $(\eta \leq 0.6)$ the fine-grained concrete works more resiliently, since its elasticity coefficients are higher than those for NW concrete by $5.2 \%$ $10.4 \%$. At higher loading levels $(\eta>0.6)$, the inverse relation is observed: NW concrete elasticity coefficients are higher by $3.5 \%-15.5 \%$ than $v$ of the fine-grained concrete. Consequently, elasticity coefficients with the increasing load in fine-grained concrete elasticity are reduced faster than in usual NW concrete. In addition, with an increase in fine-grained concrete strength, the plasticity coefficients are reduced, i.e. concrete becomes more fragile. A similar property is also observed in NW concrete.

Table 1. Strength and stress-related characteristics of fine-grained concrete in short-term loading.

\begin{tabular}{|c|c|c|c|c|c|c|c|c|c|}
\hline \multirow{2}{*}{ Series } & $\bar{R}$, & $\bar{R}_{p}$, & \multirow{2}{*}{$E_{\delta} \cdot 10^{-4}}$, & \multirow{2}{*}{$E_{\delta}^{\prime} \cdot 10^{-4}}$, & \multicolumn{5}{|c|}{$v$ at $\eta=\sigma_{\delta} / \bar{R}_{p}$} \\
\cline { 7 - 11 } & $\mathrm{MPa}$ & $\mathrm{MPa}$ & $\mathrm{MPa}$ & $\mathrm{MPa}$ & 0,4 & 0,5 & 0,6 & 0,7 & 0,8 \\
\hline \multirow{2}{*}{$\mathrm{I}$} & 15,9 & 13,5 & 1,26 & 0,96 & 0,87 & 0,76 & 0,67 & 0,57 & 0,50 \\
\cline { 2 - 8 } & 16,3 & 14,7 & 1,30 & 0,99 & 0,93 & 0,76 & 0,70 & 0,60 & 0,52 \\
\hline \multirow{2}{*}{$\mathrm{II}$} & 20,8 & 16,9 & 1,31 & 1,02 & 0,86 & 0,78 & 0,71 & 0,64 & 0,58 \\
\cline { 2 - 8 } & 25,3 & 20,0 & 1,34 & 1,14 & 0,92 & 0,85 & 0,79 & 0,74 & 0,69 \\
\hline \multirow{2}{*}{$\mathrm{III}$} & 12,0 & 9,4 & 1,55 & 1,46 & 0,92 & 0,87 & 0,77 & 0,69 & 0,53 \\
\cline { 2 - 8 } & 18,3 & 12,7 & 1,64 & 1,41 & 0,94 & 0,86 & 0,78 & 0,73 & 0,62 \\
\hline IV & 13,8 & 10,5 & 1,51 & 1,16 & 0,82 & - & 0,725 & - & 0,624 \\
\hline
\end{tabular}

Figure 1 shows the dependences of the elasticity coefficient on strength, type and age of the concrete. The elasticity and plasticity coefficients for fine-grained concrete aged 14 and 28 days were compared. With the increasing age of the concrete, its elastoplastic properties decrease. It is caused by the increase in concrete strength over time.

Figure 1, a shows the dependence of the elasticity coefficient on concrete strength. With an increase in prismatic strength from $12.7 \mathrm{MPa}$ to $20.0 \mathrm{MPa}$ by $57 \%$, the elasticity coefficient at a loading level $\eta=\sigma_{\delta} / \bar{R}_{p}=0.9$ increased by $37 \%$. Consequently, the 
elastoplastic properties of fine-grained concrete depend on the concrete age and strength and differ from the properties of NW concrete.

a)

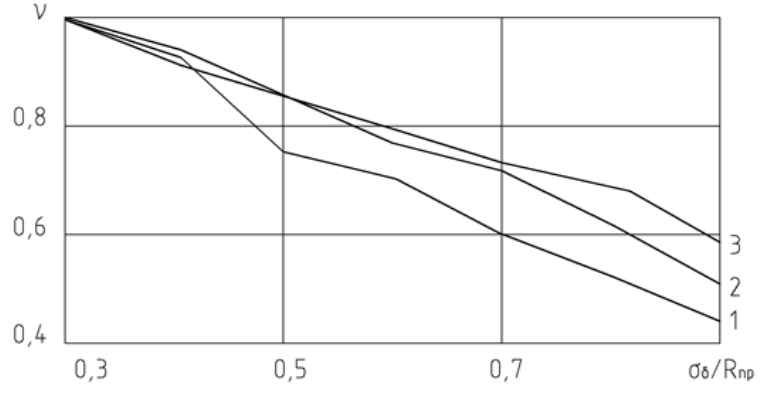

b)

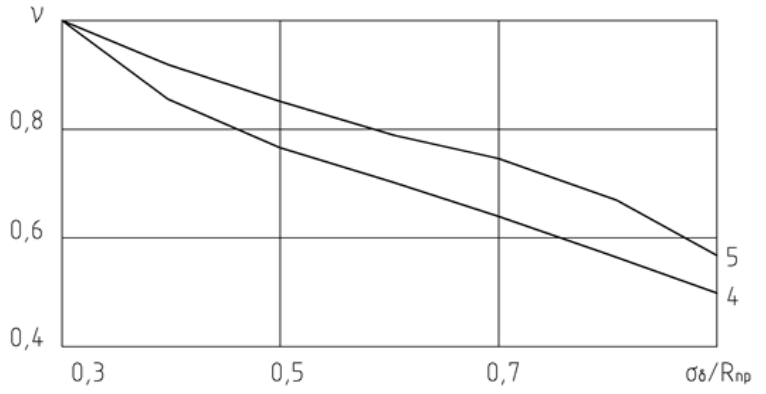

c)

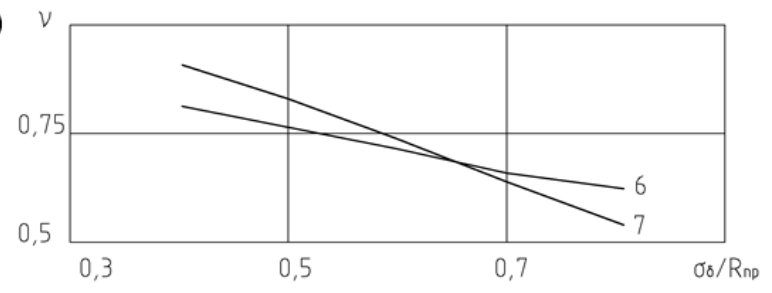

Fig. 1. Dependence of the elasticity coefficient on strength (a),

age (b), and type of concrete (c). $1-R_{p}=12,7 \mathrm{MPa}, 2-R_{\text {str }}=14,7 \mathrm{MPa}$,

3 - $R_{\text {str }}=20,0 \mathrm{MPa}, 4$ - age of 14 days, 5 - age of 28 days,

6 - normal-weight concrete, 7 - fine grain concrete.

The concrete elasticity modulus $E_{\delta}$ is to a large extent determined by strength. When normalizing the elasticity modulus of NW concrete in SP 63.13330.2012, the dependence between the average values of the concrete elasticity modulus $E_{\delta}$ and the compressive strength $\bar{R}$ is used in the form:

$$
\bar{E}_{\delta}=\frac{55000 \cdot \bar{R}}{27+\bar{R}}
$$

where $\bar{E}_{\delta}$ and $\bar{R}$ are in MPa.

The concrete elasticity modulus also depends to a large extent on its structure. Unlike conventional coarse-grained concretes, fine-grained concrete has elastic modulus about $25 \%$ lower. Considering this fine-grained concrete feature, the elastic moduli were calculated from the dependence, in which the structure of expression (8) was preserved, but $E_{\delta}$ calculated values were reduced by approximately $25 \%$ in a form: 


$$
E_{\delta}=\frac{41000 \cdot \bar{R}_{p}}{27+\bar{R}_{p}},
$$

where $E_{\delta}$ and $\bar{R}_{p}$ are given in MPa.

Experimental $\left(E_{\delta}^{\exp }\right)$ and calculated $\left(E_{\delta}^{p}\right)$ elasticity modules of fine-grained concrete are given in Table 2 .

Table 2. Results for fine-grained concrete elasticity modules under compression

\begin{tabular}{|c|c|c|c|c|c|}
\hline \multirow[b]{2}{*}{$\begin{array}{l}\bar{R}_{p}, \\
\mathrm{MPa}\end{array}$} & \multirow[b]{2}{*}{$\begin{array}{l}E_{\delta}^{\exp } \cdot 10^{-4}, \\
\mathrm{MPa}\end{array}$} & \multicolumn{4}{|c|}{ Calculated elasticity modules $E_{\delta}^{p} \cdot 10^{-4}, \mathrm{MPa}$} \\
\hline & & $\begin{array}{l}E_{\delta}^{p 1}, \\
\text { by the } \\
\text { eq. }(8)\end{array}$ & $\frac{E_{\delta}^{\mathrm{exp}}-E_{\delta}^{p 1}}{E_{\delta}^{\exp }} 100 \%$ & $\begin{array}{l}E_{\delta}^{p^{2}}, \\
\text { by the } \\
\text { eq. (9) }\end{array}$ & $\frac{E_{\delta}^{\exp }-E_{\delta}^{p 2}}{E_{\delta}^{\exp }} 100 \%$ \\
\hline 14,4 & 1,25 & 1,91 & $-52,0$ & 1,43 & $-14,4$ \\
\hline 15,9 & 1,26 & 2,04 & $-61,9$ & 1,52 & $-20,6$ \\
\hline 16,3 & 1,30 & 2,07 & $-59,2$ & 1,54 & $-18,5$ \\
\hline 16,9 & 1,40 & 2,12 & $-51,4$ & 1,58 & $-12,8$ \\
\hline 18,3 & 1,64 & 2,22 & $-35,4$ & 1,66 & $-1,2$ \\
\hline 20,8 & 1,31 & 2,39 & $-82,4$ & 1,78 & $-35,9$ \\
\hline 25,3 & 1,34 & 2,66 & $-98,5$ & 1,98 & $-47,8$ \\
\hline 27,8 & 1,62 & 2,79 & $-72,2$ & 2,08 & $-28,4$ \\
\hline \multicolumn{2}{|c|}{ average } & & $-64,1$ & & $-22,4$ \\
\hline
\end{tabular}

The important information about concrete behavior in the area close to fracture is acquired from the compression diagrams obtained with increasing load before the destruction. These diagrams, shown in Figures 2 and 3, allow for comparing the deformation characteristics of concretes of various compositions, as tests were conducted under the same conditions.

a)

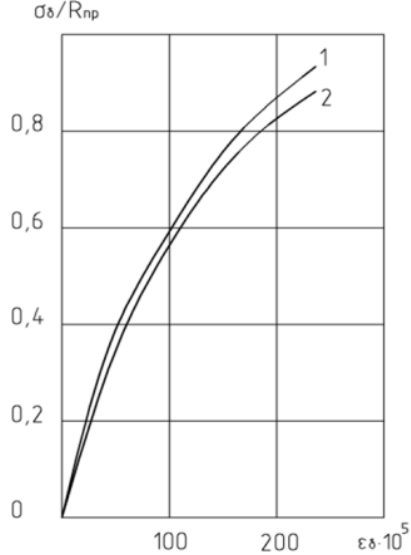

b)

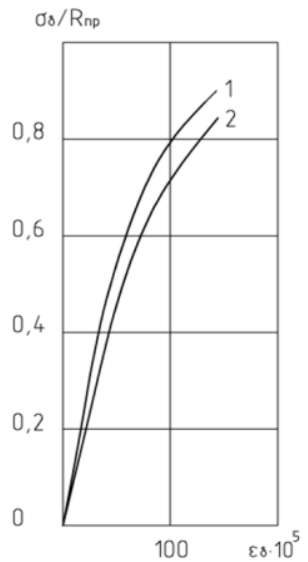

c)

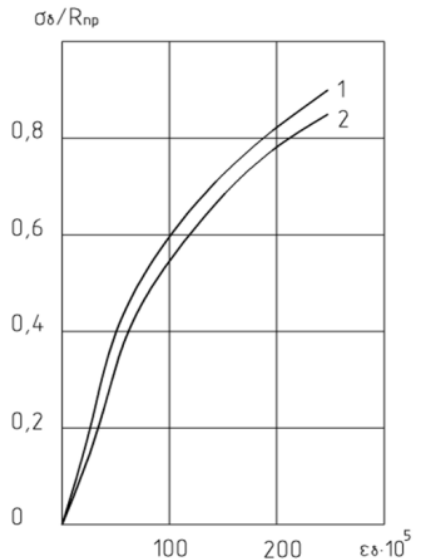

Fig. 2. Dependence $\sigma_{\delta}-\varepsilon_{\delta}$ : (a) - series II, (b) - series I, (c) - series III, 1 - age of 14 days, 2 - age of 28 days. 


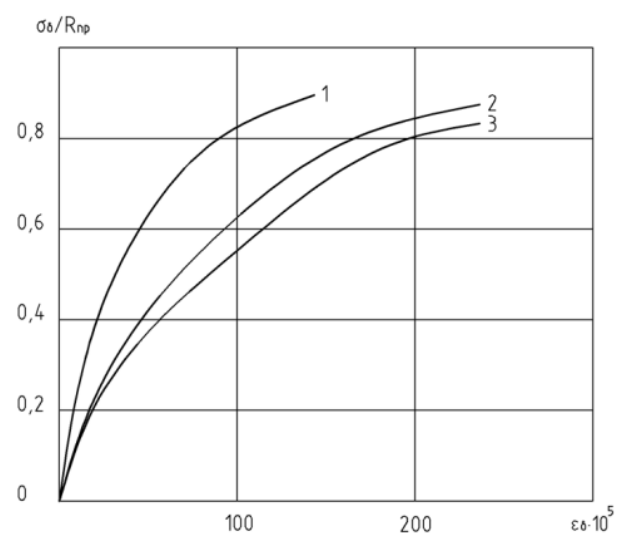

a)

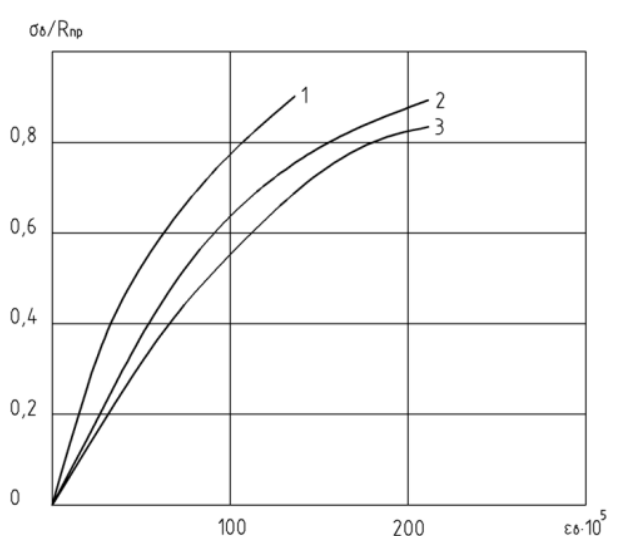

b)

Fig. 3. Dependence $\sigma_{\delta}-\varepsilon_{\delta}$. (a) - age of 14 days, (b) - age of 28 days, 1 - series III, 2 - series I, 3 - series II.

When comparing concrete deformation diagrams $\sigma_{\delta}-\varepsilon_{\delta}$ at the age of 14 and 28 days, it can be seen, that as the age of fine-grained concrete increases, the concrete becomes more brittle. The dependences of the elasticity coefficient on the concrete age given in Fig. 1, b confirm this fact.

Elasticity coefficients of the concrete at the age of 28 days at all loading levels exceeded similar coefficients of the concrete aged 14 days. In all cases, longitudinal strains at the age of 28 days was greater than the deformation of the concrete aged 14 days. It is explained by the fact that as the age of concrete increases, its compressive strength increases and the concrete works more resiliently.

It should be noted that it is about mean normal concrete strains, measured at large gauge bases, and not local ones, which can take into account local large strains due to the heterogeneity of the concrete structure.

\section{Conclusions}

One of the possible theoretical approaches related to the calculation of the reliability and accident risk for fine-grained concrete structure was set forth. Experimental studies of stress-related properties of fine-grained concrete at different ages and different loading levels were carried out. Dependences of the elasticity coefficient on strength, type and age of the concrete were assessed. Full diagrams of concrete deformation were constructed. The theoretical experimental evidence obtained can be used in the designing the load-carrying and enclosure structures made of such materials.

\section{References}

1. V.M. Bondarenko, V.I. Kolchunov, Calculated models of resistance to reinforced concrete (ASV Press, Moscow, 2004)

2. A.G. Tamrazyan, L.A. Avetisyan, MATEC Web of Conferences 86, 01029 (2016)

3. A.G. Tamrazyan, D. Popov, MATEC Web of Conferences 117, 00162 (2017)

4. I.N. Serpik, A.V. Alekseytsev, Magazine of Civil Engineering 1 (61), 14-24 (2016)

5. I.N. Serpik, A.V. Alekseytsev, P.Y. Balabin, N.S. Kurchenko, Magazine of Civil Engineering 8 (76), 181-192 (2017) 
6. K.Z. Galustov, Nonlinear theory of creep of concrete and calculation of reinforced concrete structures (Fizmatgiz, Moscow, 2006)

7. I.N. Serpik, A.V. Alekseytsev, P.Y. Balabin, Periodica Polytechnika Civil Engineering 61 (3), 471-482 (2017)

8. V.D. Raizer, Reliability of Structures: Analysis and Applications (Backbone Publishing Company, 2009)

9. Rules for allocating a budget from the reserve fund of the Government of the Russian Federation for the prevention and liquidation of emergency situations and consequences of natural disasters. As revised by the Government of the Russian Federation 2015

10. A.V. Alekseytsev, Magazine of Civil Engineering 5 (40), 28-37 (2013).

11. H.F. Arutjunjan, A.V. Mancshirov Contact problems of the creep theory (NAN RA, Moscow, 2004)

12. A.V. Alekseytsev, N.S. Kurchenko, Magazine of Civil Engineering 5 (73), 3-13 (2017)

13. Tamrazyan A.G, Avetisyan L.A., IOP Conf. Ser.: Mater. Sci. Eng., 365, 052034 (2018)

14. I.I. Ulizki, Analysis of reinforced concrete structures taking into account long time processes (Stroyizdat, USSR, 1960) 